\title{
Medical and Surgical Conditions for the Treatment of Malabsorption
}

\author{
Martina Kadmon ${ }^{a} \quad$ Kerstin Schütte $^{b} \quad$ Markus Büchler $^{\mathrm{a}} \quad$ Peter Malfertheiner $^{\mathrm{b}}$ \\ ${ }^{a}$ Department of General, Visceral, and Transplantation Surgery, University of Heidelberg, \\ ${ }^{\mathrm{b}}$ Department of Gastroenterology, Hepatology and Infectious Diseases, Otto von Guericke University of Magdeburg, Germany
}

In this edition of VisZERALMEDIZIN, we address an important medical subject in the best tradition of interdisciplinary expertise in gastrointestinal medicine and surgery, i.e. digestive insufficiency, maldigestion and malabsorption, as well as their causes, pathophysiology, and therapeutic approaches.

There are multiple causes of digestive insufficiency, and the clinical occurence is multifaceted. The small intestine and the pancreas are the key players in normal digestion. If these organs are diseased, digestive function can be significantly limited to the point of non-survivability, depending on the scope of the damage. In addition, the stomach, with its acid secretion and well-orchestrated emptying of food in cooperation with bile secretion, plays an important modulating role for a smooth digestive process. Radical surgical methods as part of oncological interventions and repair procedures in patients with Crohn's disease that is refractory to treatment add to the various causes of maldigestion or malabsorption. Fortunately, we can turn to effective measures nowadays to cure or compensate for malabsorption.
Modern surgical methods have become established for the treatment of short bowel syndrome, and surgical procedures in the upper gastrointestinal tract and pancreas lay claim to maximally organ-protecting interventions for the preservation of cibal synchrony.

In this special issue, experts with long-standing clinical experience and detailed fundamental knowledge contribute extensively to the topic of malabsorption. New findings and treatment methods have emerged, particularly in the field of celiac disease, and there are also new aspects regarding Whipple's disease which are successfully being incorporated in the treatment of this disease.

Today, in the era of the obesity epidemic and the associated success story of bariatric surgery, malabsorption can also occur deliberately in subtle clinical forms. These aspects are also taken into account to avoid jeopardizing the advantages of metabolic corrections through bariatric surgery by means of 'micronutrient malabsorption'.

With this edition of Viszeralmedizin, we thus hope to present a special issue that is equally fascinating for gastroenterologists and surgeons alike.

\section{KARGER}

Fax +497614520714

Information@Karger.com

www.karger.com
Prof. Dr. med. Peter Malfertheiner

Department of Gastroenterology, Hepatology and Infectious Diseases

Otto von Guericke University of Magdeburg

Leipziger Straße 44, 39120 Magdeburg, Germany

peter.malfertheiner@med.ovgu.de 